\title{
Wind Tunnel Test Research on Aerodynamic Characteristics of Iced Conductors
}

\author{
$\mathrm{Li} \mathrm{Li}^{1}$, Guo Li ${ }^{1,}$,, Huajin $\mathrm{Cao}^{2}$ \\ ${ }^{1}$ School of Civil Engineering \& Mechanics, Huazhong University of Science and Technology, Wuhan, P. R. China \\ ${ }^{2}$ China Railway Siyuan Survey and Design Group Co., Ltd., Wuhan, P. R. China
}

\section{Email address:}

13476294116@163.com (Li Li), li_guo@hust.edu.cn (Guo Li), 17671671516@163.com (Huajin Cao)

*Corresponding author

\section{To cite this article:}

Li Li, Guo Li, Huajin Cao. Wind Tunnel Test Research on Aerodynamic Characteristics of Iced Conductors. Advances in Applied Sciences. Vol. 5, No. 1, 2020, pp. 11-19. doi: 10.11648/j.aas.20200501.12

Received: December 5, 2019; Accepted: December 12, 2019; Published: April 1, 2020

\begin{abstract}
Aiming at that the wind tunnel tests on aerodynamic characteristics of iced conductors were not systematic enough, the wind tunnel test for different icing types was conducted to study the aerodynamic characteristics of iced conductors more systematically. It can accounts for the characteristics of structure and wind field. The aerodynamic characteristics of different icing types such as crescent, fan and corona shapes with different icing thickness were obtained, and the effect of mean wind speed, turbulence and two-dimensional flow on aerodynamic characteristics were also investigated. It is concluded that the icing shapes have much effect on aerodynamic characteristics. The aerodynamic characteristics of rigid segment model with crescent-shaped are regular, while these of corona-shaped and fan-shaped have more peaks and troughs. In addition, the aerodynamic instability of iced conductors is more pronounced in turbulence. Furthermore, the wind speed has significant effects on drag coefficient, but the effects on lift coefficient is little.
\end{abstract}

Keywords: Galloping, Aerodynamic Characteristic, Wind Tunnel Test, Iced Conductor

\section{Introduction}

Galloping is a self-excited instability related to slender structures with non-circular cross-sections. Appearing with low frequency and large amplitude, galloping of transmission lines may cause electric accident, e.g., flashover, short circuit, line break and tower collapse [1]. Therefore, it is very important to study galloping and anti-galloping means for the safe operation of the power grid. Since galloping of iced conductors is one type of wind-induced vibration owing to aerodynamic instability, research on aerodynamic characteristics of iced conductors is essential to solve the matters. The wind tunnel test is an important means for aerodynamic research, and many wind tunnel experiments have been carried out since galloping was discovered. Loredo-Souza and Davenport [2] proposed a method to design aero-elastic model of a transmission line for wind tunnel test. The stability of the sectional models of bundle conductors mounted by springs in wind tunnel were analyzed by Wardlaw et al. [3]. Weaver and Veljkovic [4] performed a wind tunnel experiment to investigate the vortex shedding and galloping of open semi-circular and parabolic cylinders in cross-flow. The aerodynamic mechanism of torsional flutter of bluff structures was investigated by Nakamura [5] through experimental and theoretical means. By means of wind tunnel tests, Alonso and Meseguer [6] analyzed the transverse galloping stability of triangular cross-section bodies and found that the stability depended on both cross-sectional geometry and angle of attack. Alonso et al. [7] also analyzed the transverse galloping stability of biconvex and rhomboidal cross-section and revealed how the aerodynamic characteristics of the bodies evolved when the geometry of cross-section changed. The galloping of lightly-iced transmission lines was studied through a series of wind tunnel experiments by Fleming and Popplewell [8]. Bo Yan et al. [9] carried out wind tunnel tests of quad bundle conductor segments with crescent-shaped to investigate the galloping characteristics of iced quad bundle conductors. Gandia et al. [10] presented static and dynamic 
wind tunnel experiments, which analyzed the galloping Stability of Porous H-section beams. W. Lou et al. [11] carried out wind tunnel test to study the galloping of an ice-coated D-shape sectional model of 6-bundled conductors. M. Li [12] measured the drag coefficient of overhead conductor with a camber concave shape and ACSR by wind tunnel test. $\mathrm{C}$. Wu et al. [13] investigated the variation of aerodynamic coefficients with wind attack angle for twin bundle conductors in wind tunnel. You Yi et al. [14] conducted wind tunnel test to study the aerodynamic characteristics of crescent and D-shape iced conductor with different wind velocities, wind attack angles and torsional elastic support stiffness.

In summary, researches on galloping by wind tunnel tests have so far focused on aerodynamic characteristics of a particular section, and the wind tunnel tests of iced conductors are still not system and rich enough. Therefore, it is essential to study the aerodynamic characteristics of iced conductors more systematically and deeply.

This paper thus presents a force measurement test of segment models to study the aerodynamic characteristics of iced conductors. In section 2 , the theory of the aerodynamic characteristics of iced conductors are introduced. Section 3 presents the details of the test, including similar conditions and working conditions. Section 4 gives the results of the experiment that the effects of icing shape, ice thickness, mean wind speed, turbulence and boundary layer on aerodynamic characteristics. Finally, the results are discussed and summarized in section 5 .

\section{Concept of the Aerodynamic Characteristics of Iced Conductor}

\subsection{Icing Shape and Icing Thickness}

The shape of iced conductors is generally not round because of the wind direction and torsional rigidity of conductors. The general shapes are those of crescent (X), fan (S) and corona $(\mathrm{G})$, as shown in Figure 1, and the icing thickness has a wide range. Therefore, the paper focused on the three different icing shapes under different icing thickness. Especially, the icing thickness is the thickness of the ice in the diameter direction, as shown in the Figure 2. And $\lambda_{i+}$ is used as a measure of icing thickness, which is expressed as follows:

$$
\lambda_{i+}=(d+D) / D=(D+) / D \quad i=X, S, G
$$

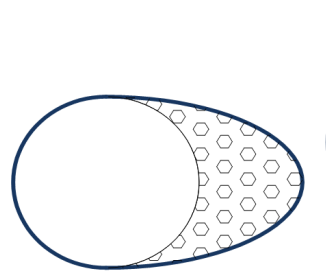

(a) Crescent shape

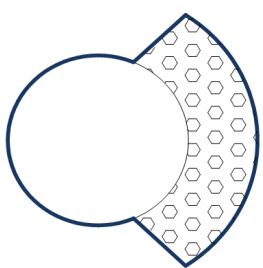

(b) Fan shape

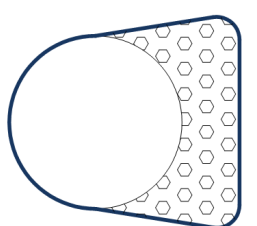

(c) Corona shape
Figure 1. Icing shapes of iced transmission conductors.

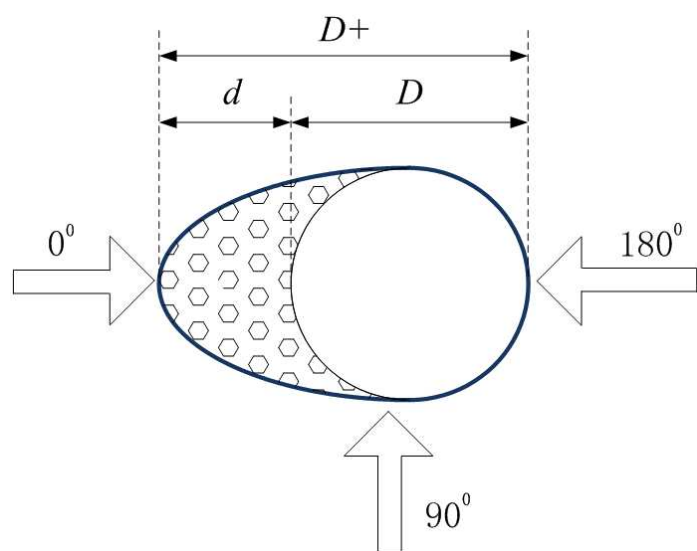

Figure 2. Icing thickness and wind direction angle setting of iced transmission conductor.

\subsection{Aerodynamic Coefficient}

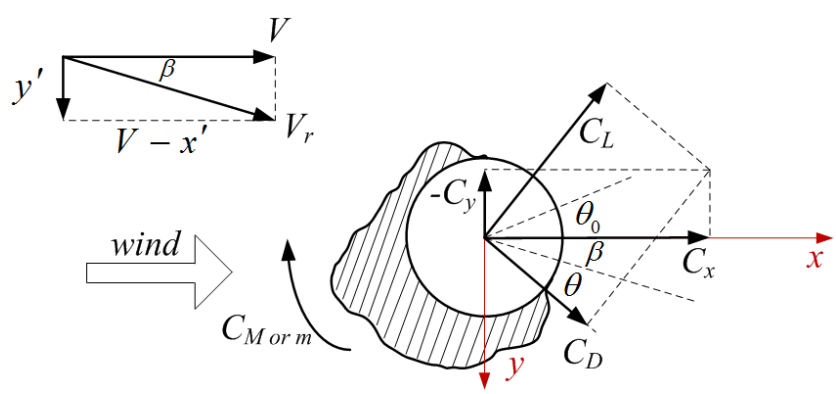

Figure 3. Aerodynamic coefficient definitions of iced transmission conductors.

This paper focuses on the aerodynamic characteristics of iced conductors with different icing types and icing thicknesses to explore the mechanism of galloping, so the aerodynamic characteristics are studied based on a three degree of freedom oscillator. There are three aerodynamic forces in three different directions, which are the drag force $F_{x}$ along the direction of the wind speed, lift force $F_{y}$ perpendicular to the direction of the wind speed and torque force $F_{m}$ in the torsional direction. According to the quasi-steady theory, the aerodynamic force of unit length in three different directions can be expressed as

$$
\left\{\begin{array}{l}
F_{y}=\frac{1}{2} \rho V_{r}^{2} D C_{y} \\
F_{x}=\frac{1}{2} \rho V_{r}^{2} D C_{x} \\
F_{m}=\frac{1}{2} \rho V_{r}^{2} D^{2} C_{m}
\end{array}\right.
$$

where $\rho$ is the air density, $C_{x}$ is the horizontal aerodynamic coefficient, $C_{y}$ is the vertical aerodynamic coefficient and $C_{m}$ is the torsional aerodynamic coefficient. In mechanical analysis, it is reasonable to treat the average force in the stable region as the aerodynamic force of iced conductor, because the vibration frequency of iced conductor is much smaller than the vortex frequency. 
Especially, there are also three aerodynamic coefficients which are the drag coefficient $C_{\text {drag }}$ along the direction of the wind speed, lift coefficient $C_{\text {lift }}$ perpendicular to the direction of the wind speed and torque coefficient $C_{\text {moment }}$ in the torsional direction and different from $C_{x}, C_{y}$ and $C_{m}$. The relationship of them are shown in Figure 3. Then $C_{x}, C_{y}$ and $C_{m}$ are derived as follows:

$$
\left\{\begin{array}{l}
C_{y}\left(\alpha_{R}\right)=C_{D}\left(\alpha_{R}\right) \cdot \sin \alpha-C_{L}\left(\alpha_{R}\right) \cdot \cos \alpha \\
C_{x}\left(\alpha_{R}\right)=C_{D}\left(\alpha_{R}\right) \cdot \cos \alpha+C_{L}\left(\alpha_{R}\right) \cdot \sin \alpha \\
C_{m}\left(\alpha_{R}\right)=C_{M}\left(\alpha_{R}\right)
\end{array}\right.
$$

where $\alpha$ is the attack angle of force decomposition and $\alpha_{R}$ is the wind angle of attack, which are expressed as follows:

$$
\begin{gathered}
V_{r}=\sqrt{\left(V-x^{\prime}\right)^{2}+y^{\prime 2}} \\
\beta=\arctan \left(\frac{y^{\prime}}{V-x^{\prime}}\right) \approx \frac{y^{\prime}}{V-x^{\prime}},\left(y^{\prime}<<V-x^{\prime}\right) \\
\alpha=\beta+\theta
\end{gathered}
$$

$$
\alpha_{R}=\theta_{0}+\alpha
$$

where $y$ is the vertical displacement of conductor, $y^{\prime}$ is the vertical velocity of conductor, $x^{\prime}$ is the horizontal velocity of conductor, $V$ is the horizontal wind speed, $\theta_{0}$ is the initial wind angle of attack and $\theta$ is the torsion angle of conductor.

\section{Wind Tunnel Test}

This wind tunnel test is conducted in the first test section of the wind tunnel laboratory in Shantou University. The wind tunnel is shown in Figure 4. The force measurement test is the measure of overall wind load (including lift force, drag force and overturning moment) acting on the model which is fixed on the admeasuring apparatus. Figure 5 shows the six component force measuring balance, DELTA, used in this test. The rated range and resolution, respectively, are $330 \mathrm{~N}$ and $1 / 64 \mathrm{~N}$ in horizontal direction, $990 \mathrm{~N}$ and $1 / 32$ in vertical direction, $30 \mathrm{Nm}$ and $3 / 3200 \mathrm{Nm}$ for bending moment and torque. Figure 6 shows the segment models of iced conductors with different icing shape.

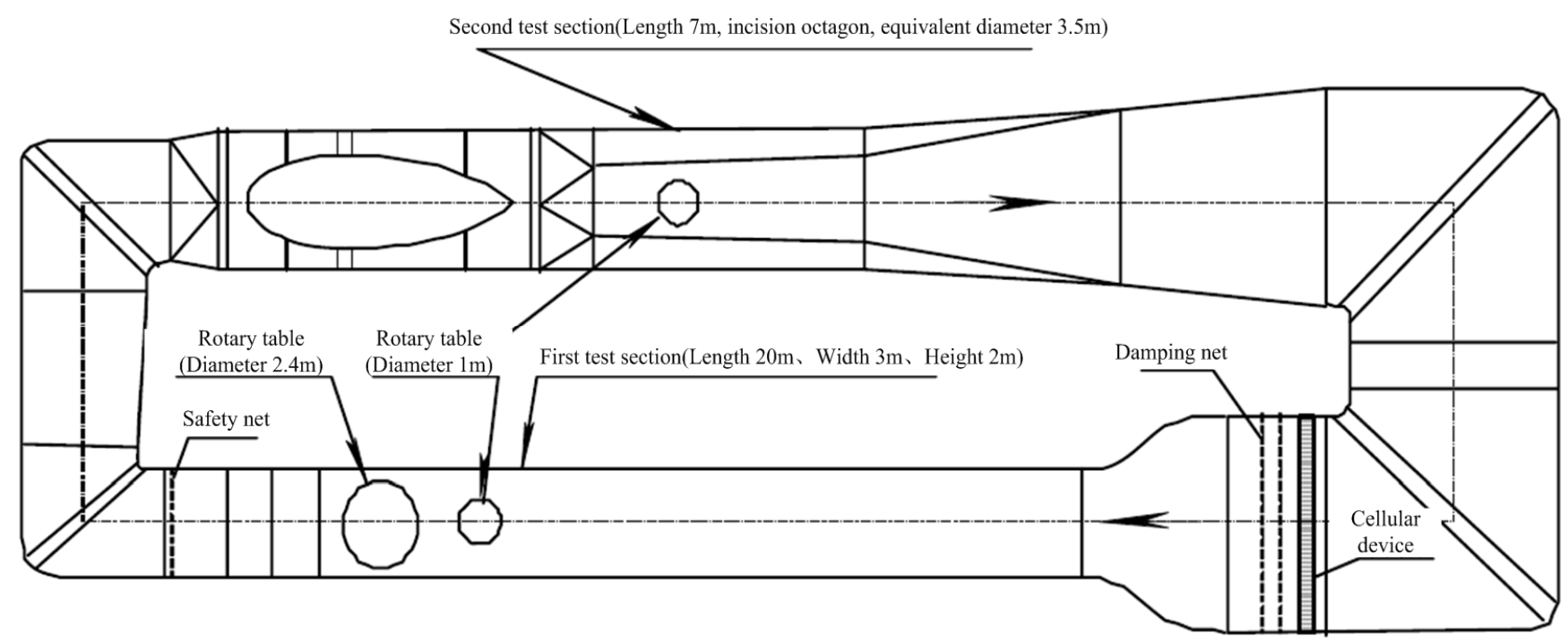

Figure 4. Wind tunnel laboratory structure of Shantou University.
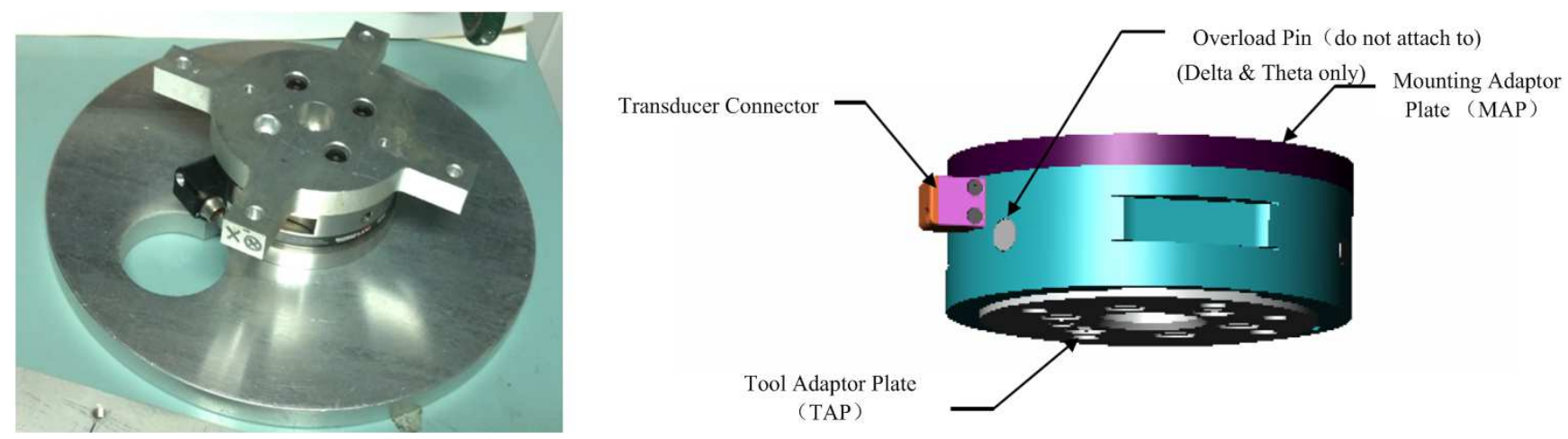

Figure 5. Force measuring balance and DELTA sensors. 


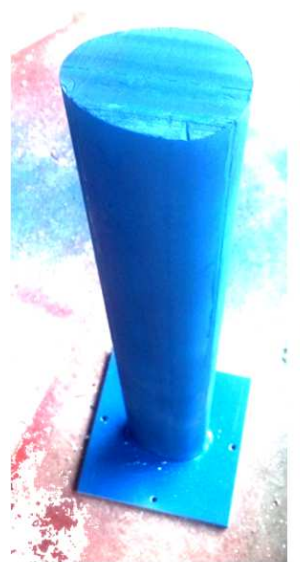

(a) Crescent model

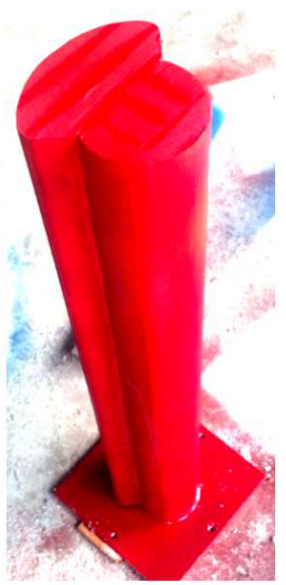

(b) Fan model

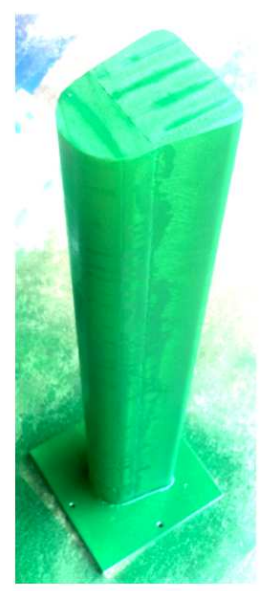

(c) Corona model
Figure 6. Segment models of the iced conductors with different icing shape.

\subsection{Similar Conditions}

According to the theory of wind tunnel experiment [15], the test should satisfy the similar conditions.

\subsubsection{SNR (Signal to Noise Ratio)}

To ensure the wind load acting on model is large enough, the diameter of the model is 2 times the diameter of actual conductor, which is $D_{\text {full }} / D_{\text {model }}=1 / 2$, so that the measurement results have sufficient SNR. The diameter of actual conductor is $30 \mathrm{~mm}$, so the diameter of the model is $60 \mathrm{~mm}$.

\subsubsection{Model Rigidity}

The whole vibration system consists of the force measurement system and the test model. Figure 7 shows the vibration characteristic curve of the system. There is no problem when only the average wind load is chosen as the measuring object. But to measure the fluctuating component, we must ensure that the frequencies of the measuring range is not in the resonance range of the balance-model vibration system. In order to measure the wind load generated only by the wind, it is necessary to improve the stiffness and reduce the weight of measurement system so that the test models are rigid enough. Figures 8 and 9 show that the test natural frequencies of the typical model for tapping test and spectrum analysis, which are far higher than the concerned frequency range of aerodynamic force.

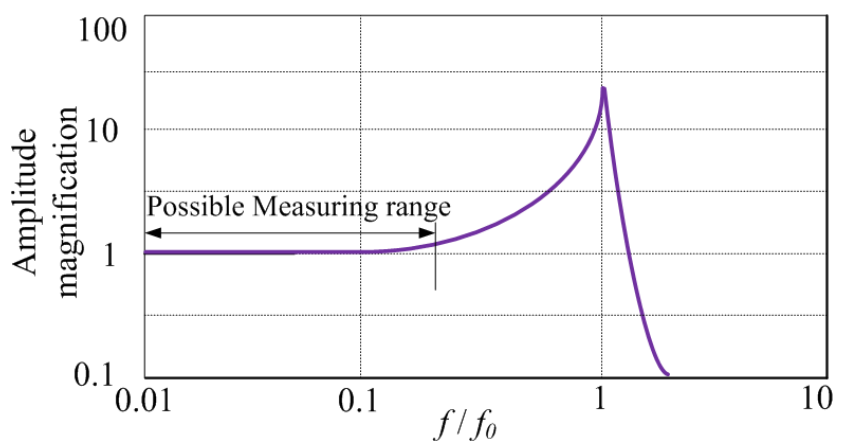

Figure 7. Vibration characteristics measurement of the system.

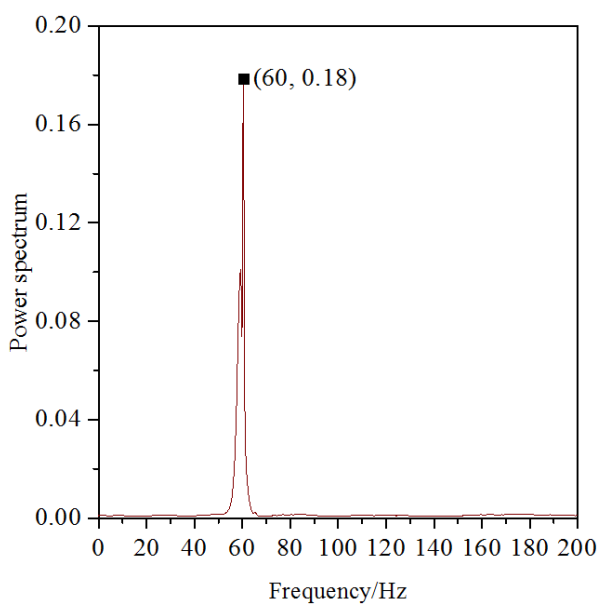

Figure 8. Power -frequency relationship with icing shape coefficient of $\lambda_{G+}=1.4$

\subsubsection{Reynolds Number}

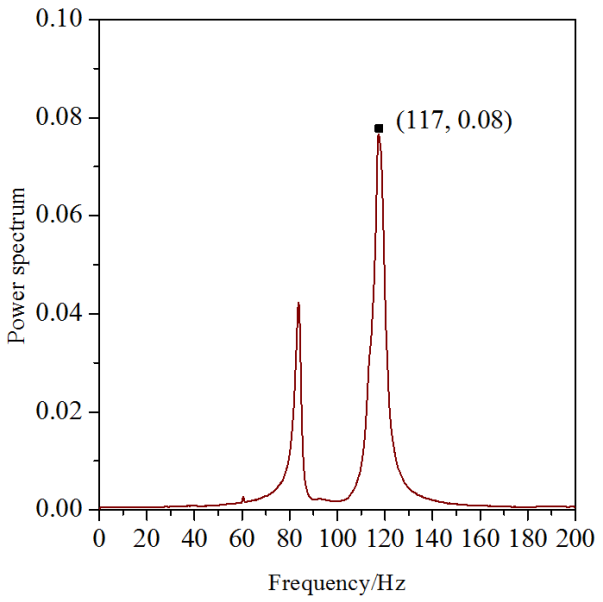

Figure 9. Power-frequency relationship with icing shape coefficient of $\lambda_{S+}=1.2$.

Reynolds number is the ratio of fluid inertia force to viscous force, which can be expressed as

$$
\operatorname{Re}=V D / v
$$

where $v$ is the dynamic viscosity coefficient of fluid.

To satisfy the similar conditions, the Reynolds number of the test fluid should be same to that of natural wind:

$$
(V D / v)_{\bmod e l}=(V D / v)_{\text {full }}
$$

Due to the temperature and pressure of the fluid in wind tunnel test is same with natural wind, the dynamic viscosity coefficients of natural wind and the wind tunnel flow are the same.

As $D_{\text {full }} / D_{\text {model }}=1 / 2$ is used in this test, the ratio of the wind speed in wind tunnel to the actual wind speed is

$$
V_{\text {model }} / V_{\text {full }}=D_{\text {full }} / D_{\text {model }}=1 / 2
$$

So the test wind speed is 0.5 times of natural wind speed, taking $22 \mathrm{~m} / \mathrm{s}$ as natural wind speed and $11 \mathrm{~m} / \mathrm{s}$ as the test 
wind speed.

Figure 10 shows the Cobra 220 probe for the measurement of wind speed in the test. Figure 11 shows the grid layout for generating turbulence. Figure 12 shows the time history of fluctuating wind used in the test, with the average wind speed being $11 \mathrm{~m} / \mathrm{s}$ and the turbulence intensity being $8 \%$. Figure 13 shows the time history of the uniform flow used in the test. All the above data are measured by a Cobra 220 probe.

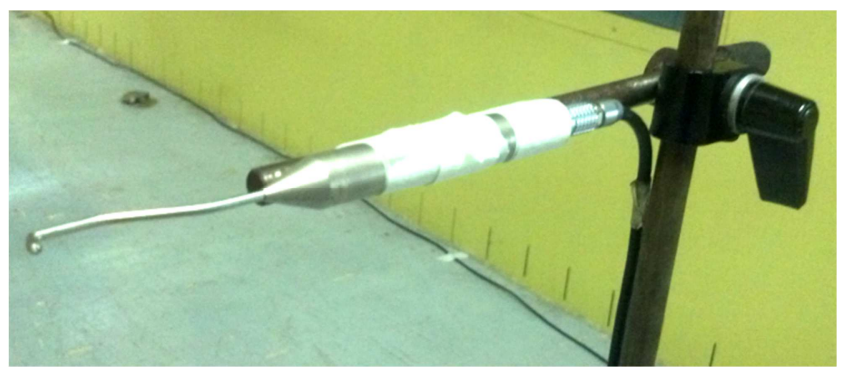

Figure 10. Cobra probe for wind speed measurement.

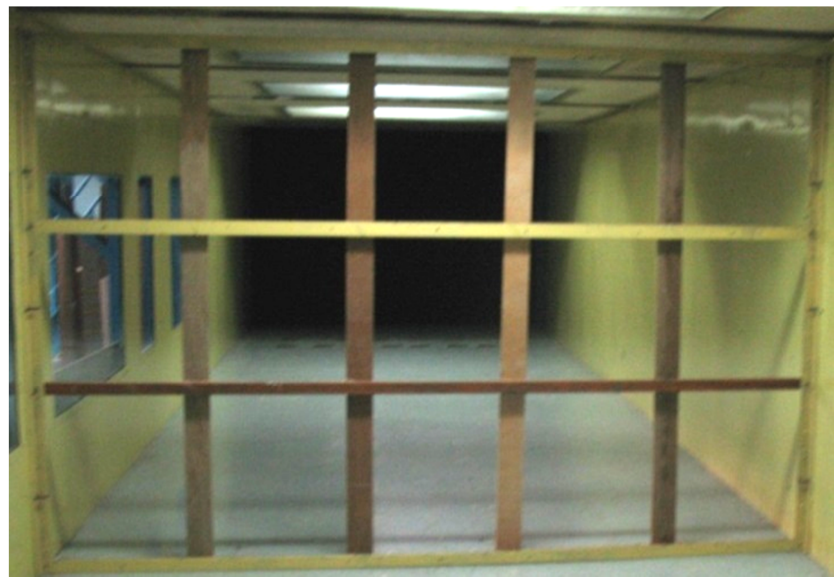

Figure 11. Grid layout.

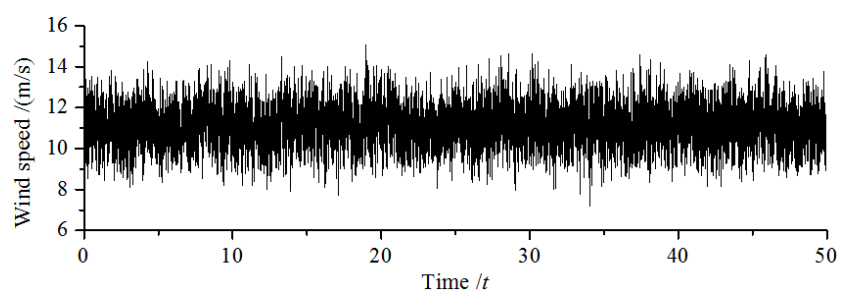

Figure 12. Time history of the fluctuating wind with the average wind speed of $11 \mathrm{~m} / \mathrm{s}$ and the turbulence intensity of $8 \%$.

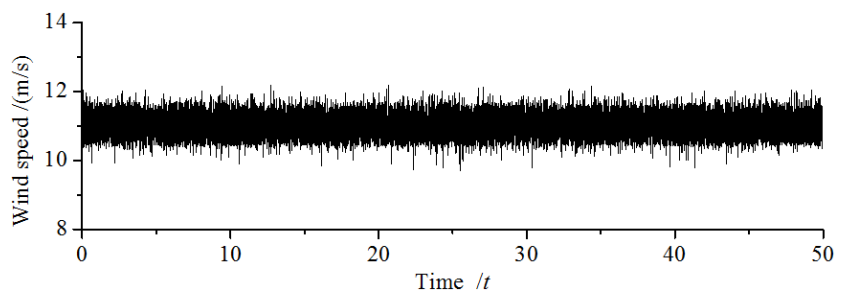

Figure 13. Time history of the uniform flow with the average wind speed of 11 $\mathrm{m} / \mathrm{s}$.

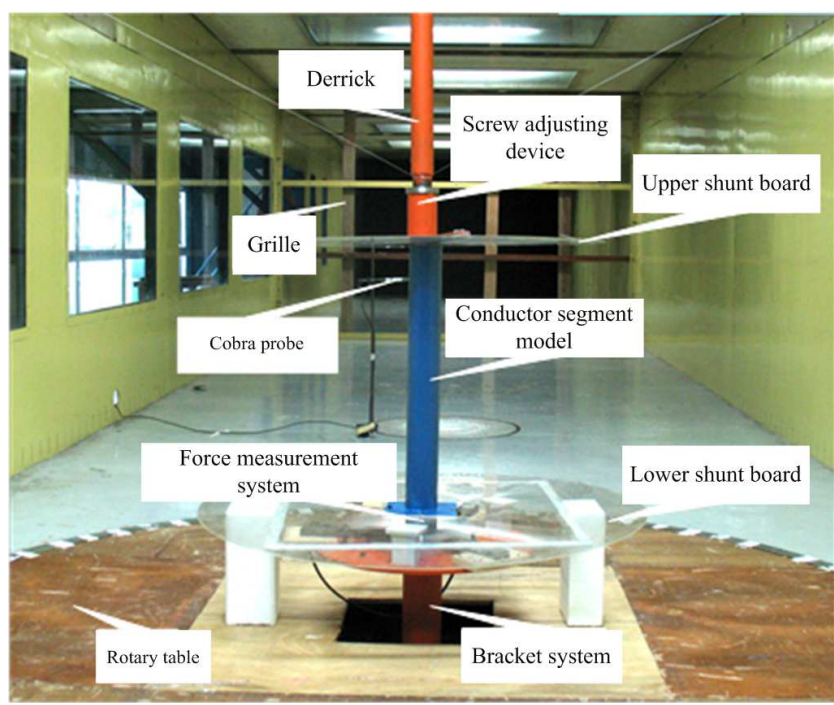

Figure 14. Force measurement test for iced conductor segment model.

\subsubsection{Boundary Layer Wind Speed}

Because of the influence of boundary layer friction, the wind speed in the wind tunnel is unstable near the boundary layer, but the boundary layer effect does not exist for the overhead conductors. In order to reduce the effect of boundary layer friction, the bottom bracket system is designed to ensure that the distance between the segment model and the ground is long enough. The distance is $22 \mathrm{~cm}$ in the test, which is completely out of the unstable wind speed zone. Moreover, the segment model is long enough. But if the segment model is too long, its stiffness decreases, which may cause vibration of the model. Therefore, the length of the segment model is $60 \mathrm{~cm}$.

To ensure the two-dimensional feature of the flow field, two shunt boards are set on both ends of the segmental model. The diameters of the lower and upper shunt boards are $100 \mathrm{~cm}$ and $80 \mathrm{~cm}$ respectively. The derrick and screw device were also designed to ensure the accuracy of aperture, as shown in Figure 14.

\subsection{Working Conditions of the Wind Tunnel Test}

Working conditions in the paper are designed as follows:

Condition 1: Force measurement experiment for the segment model of circular conductor is conducted in uniform flow with the wind speed of $7.5 \mathrm{~m} / \mathrm{s}$.

Condition 2-4: Force measurement experiments for the segment models of iced conductor with different icing shape coefficients $\left(\lambda_{X^{+}}=1.4, \lambda_{S+}=1.4\right.$ and $\left.\lambda_{G+}=1.4\right)$ are conducted in uniform flow with the wind speed of $11 \mathrm{~m} / \mathrm{s}$ at high attack angle from 0 degree to 180 degree.

Condition 5: Force measurement experiments for the segment model of iced conductor with the icing shape coefficient of $\lambda_{G+}=1.4$ shown in Figure 15(a) are conducted in uniform flow with wind speed of $7.5 \mathrm{~m} / \mathrm{s}$ at high attack angle from 0 degree to 180 degree.

Condition 6: Force measurement experiments for the segment model of iced conductor without shunt boards are conducted in uniform flow with the wind speed of $11 \mathrm{~m} / \mathrm{s}$ and 
the icing shape coefficient of $\lambda_{X^{+}}=1.4$ at high attack angle from 0 degree to 180 degree, as shown in Figure 15(b).

Condition 7-9: Force measurement experiments for the segment models of iced conductor with different icing shape coefficients $\left(\lambda_{X^{+}}=1.4, \lambda_{S^{+}}=1.2\right.$ and $\left.\lambda_{G^{+}}=1.2\right)$ are conducted in turbulence with the average wind speed of $11 \mathrm{~m} / \mathrm{s}$ and the turbulence intensity of $8 \%$ at high attack angle from 0 degree

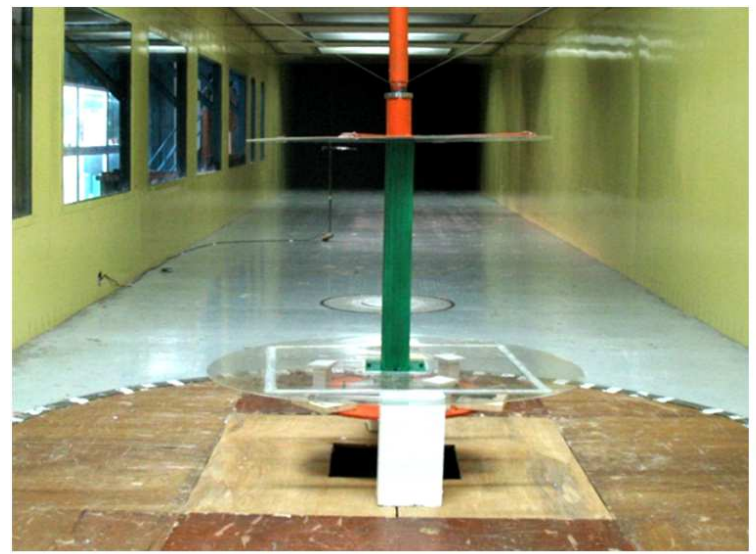

(a) Working condition 5 to 180 degree.

Condition 10: Force measurement experiments for the segment model of iced conductor with the icing shape coefficient of $\lambda_{G^{+}}=1.2$ are conducted in turbulence with the average wind speed of $7.5 \mathrm{~m} / \mathrm{s}$ and the turbulence intensity of $8 \%$ at high attack angle from 0 degree to 180 degree.

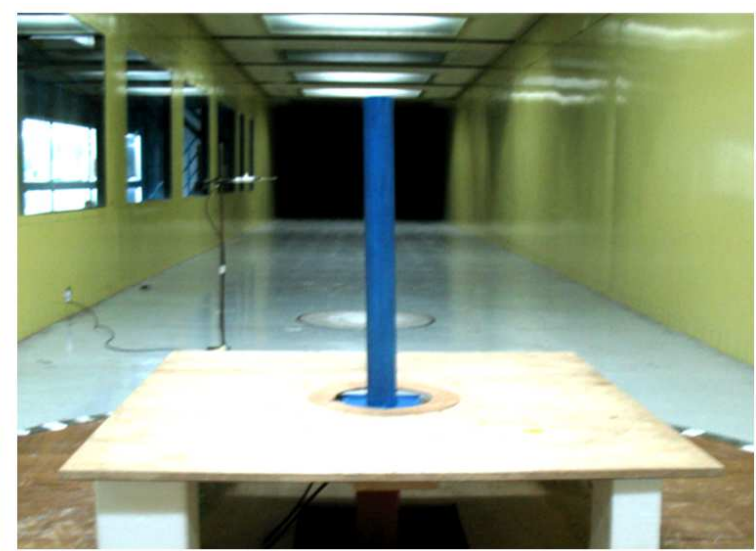

(b) Working condition 6

Figure 15. Aerodynamic tests of the segment models of iced conductor in uniform flow.

\section{Results of the Aerodynamic Test and Discussion}

The working condition 1 shown in Figure 16 is designed to validate the results of other working conditions. The segment model is a circular tube with the diameter of $50 \mathrm{~mm}$. The design wind speed in the wind tunnel is $7.5 \mathrm{~m} / \mathrm{s}$, and the Reynolds number is $2.5 \times 10^{4}$. The tested drag coefficient is 1.18 in the paper. According to paper published by Franke and Frank [16], the drag coefficient is 1.20 . Thus the accuracy of the following experiment results can be verified.

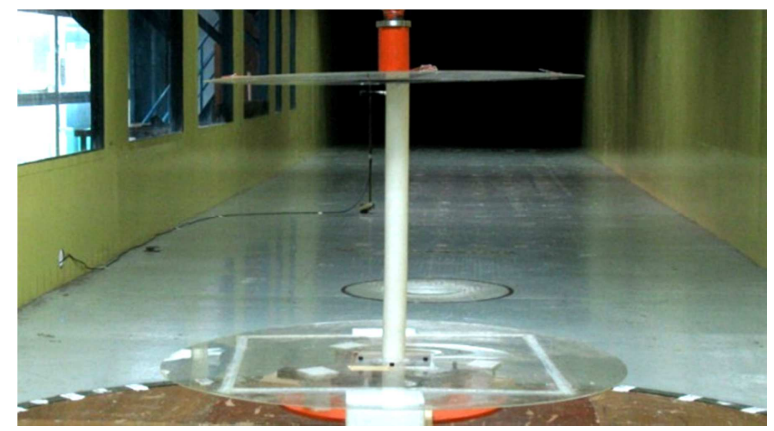

Figure 16. Wind tunnel test of the verification working condition.

\subsection{Effect of Icing Shapes on the Aerodynamic}

The aerodynamic coefficients of the models with different ice shapes were measured in wind tunnel. It can be seen from Figures 17 and 18 that the aerodynamic characteristics of the crescent model with the same icing shape coefficient are more regular in uniform flow, while the aerodynamic characteristics of the fan and corona models have more peaks and troughs.
Because the conductors with crescent-shaped ice belong to the similar streamline body and the edges and corners of the conductors with fan and corona shaped ice are relatively distinct, the aerodynamic characteristics of crescent shape differ from those of fan and corona shapes.

For the crescent model, the drag coefficient is minimum when the wind angle of attack is 0 degree or 180 degree, and it is maximum when the wind angle of attack is 90 degree. The maximum value of the drag coefficient is 1.7 , and the variation curve of the drag coefficient with the wind angle of attack displays half wave. The lift coefficient is also the minimum when the wind angle of attack is 0 degree or 180 degree, close to zero, and it has a peak and trough with the value of 0.4 and -0.5 , displaying a wave.

For the fan model, the drag coefficient reaches the maximum of 2.1 at 0 degree, and it has a peak with the value of 1.9 at 140 degree. The minimum value of the drag coefficient is 1.2 , which occurs when the wind angle of attack is 40 degree. The lift coefficient has two peaks at 30 degree and 170 degree and a trough at 110 degree. The variation curve of the lift coefficient with the wind angle of attack displays $3 / 2$ waves.

For the corona model, the maximum value of the drag coefficient is 2.0 at 0 degree or 120 degree, the minimum value is 1.0 at 180 degree, and it also has two troughs at 20 degree and 80 degree. Similar to the fan model, the variation curve of the drag coefficient with the angle for the corona model also displays $3 / 2$ waves. The maximum values appear at 20 degree and 170 degree, which are 1.1 and 1.2 respectively, and the minimum value is -1.0 at 180 degree.

The Figures 19 and 20 present the test results for different icing shapes under the action of turbulent flow with the same icing shape coefficient of 1.2. 
For the fan model, the drag coefficient reaches the maximum value of 1.9 at 0 degree, and it has peaks at 30 degree and 170 degree. The minimum value of the drag coefficient is 1.0 when the wind angle of attack is 40 degree, and the variation curve of the drag coefficient with the angle displays $3 / 2$ waves. The lift coefficient is minimum when the wind angle of attack is 100 degree or 110 degree, and it has two peaks at the degree of 30 and 150, the value of which is about 0.7 , and the variation curve of the lift coefficient with the angle also displays $3 / 2$ waves.

For the corona model, the drag coefficient is maximum when the wind angle of attack is 0 degree or 130 degree, and its minimum value is 0.9 at 100 degree. It also has a trough at 80 degree. The lift coefficient has the same variation with the fan icing shape, and the maximum values are 1.1 and 1.2 at 20 degree and 160 degree, while the minimum value appears at 90 degree.

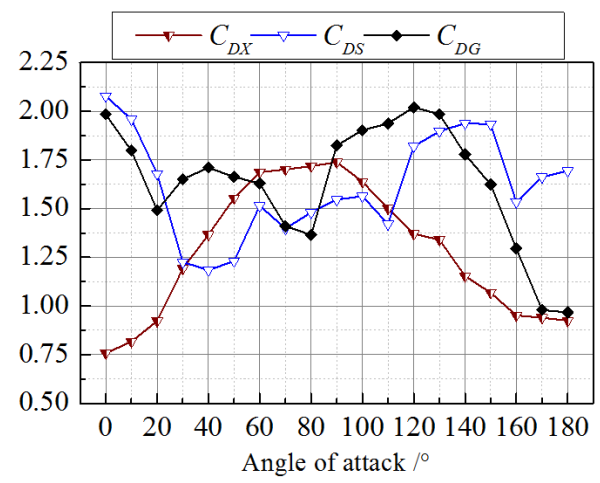

Figure 17. Drag coefficient of different icing shapes in uniform flow.

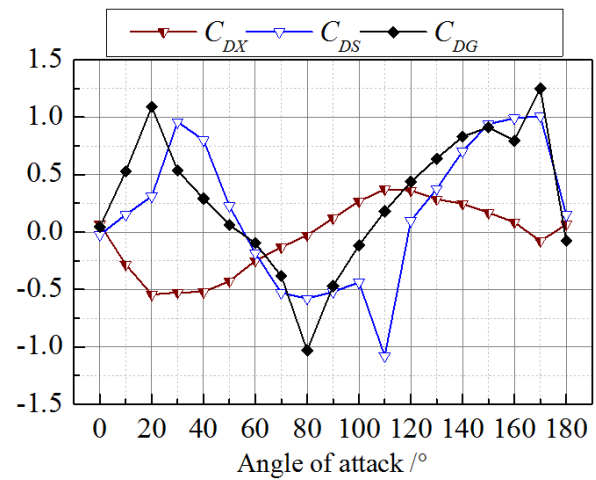

Figure 18. Lift coefficient of different icing shapes in uniform.

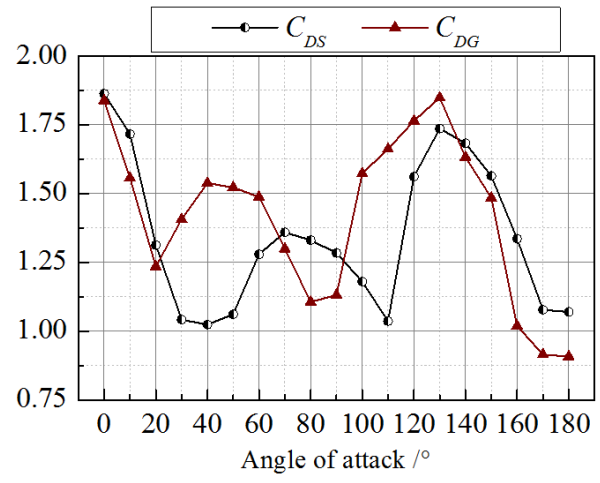

Figure 19. Drag coefficient of different icing shapes in turbulent flow.

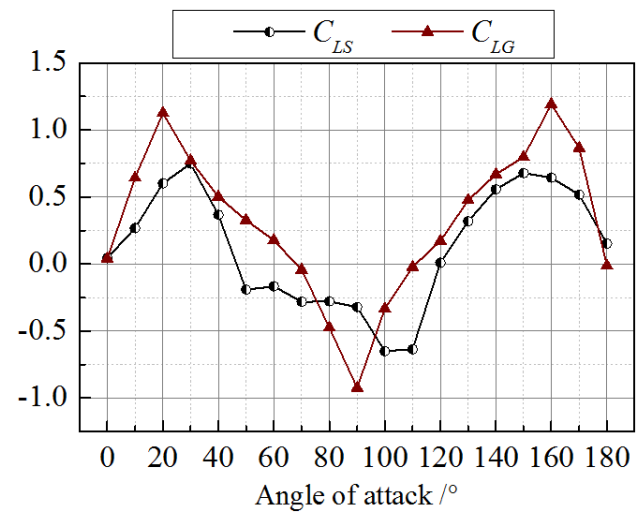

Figure 20. Lift coefficient of different icing shapes in turbulent flow.

\subsection{Effect of Two-dimensional Flow Effect on the Aerodynamic}

Figures 21 and 22 show the test results of the iced conductor, the icing shape coefficient of which is $\lambda_{X^{+}}=1.4$, with or without shunt boards. It can be seen that for the corona model, the aerodynamic coefficients of the iced conductor with shunt boards are larger than that without shunt boards in uniform flow. Moreover, the shunt board has little effect on the lift coefficient, but it has much effect on the drag coefficient. Without shunt boards, the maximum value would decreases from 1.7 to 1.4. These results indicate that the shunt boards can ensure the two-dimensional feature of the flow field and the shunt boards are indispensable to the test.

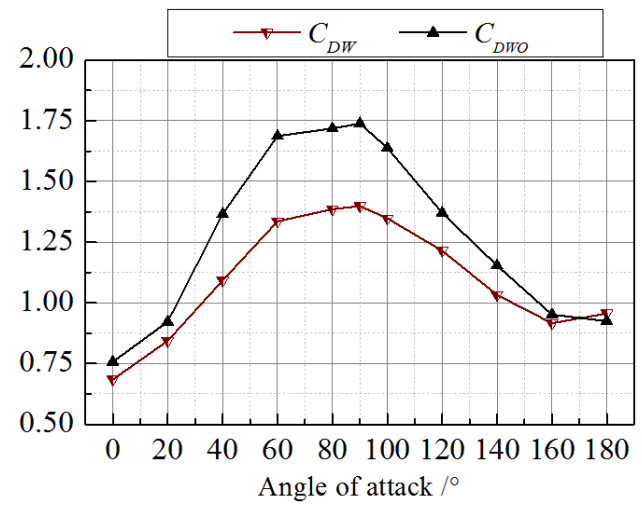

Figure 21. Drag coefficient with/without shunt boards in uniform flow.

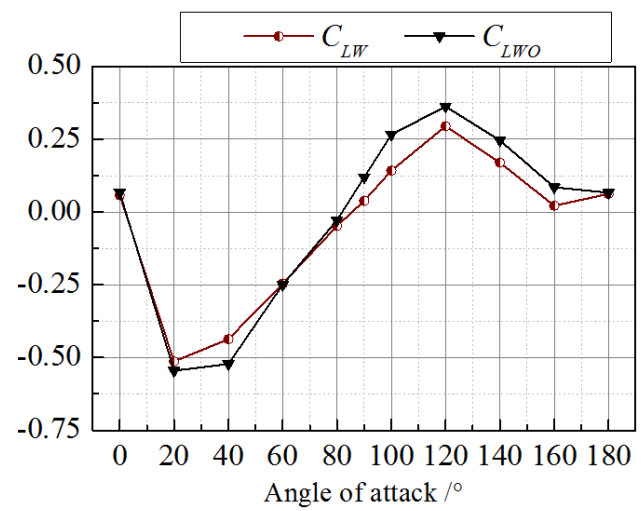

Figure 22. Lift coefficient with/without shunt boards in uniform flow. 


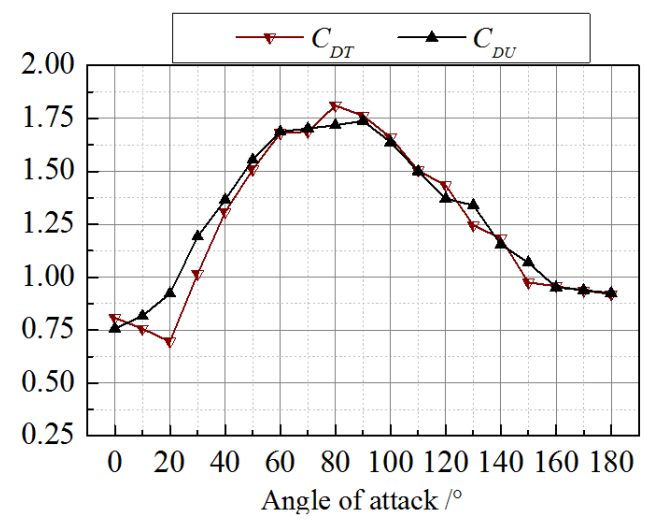

Figure 23. Drag coefficient in uniform flow and turbulent flow.

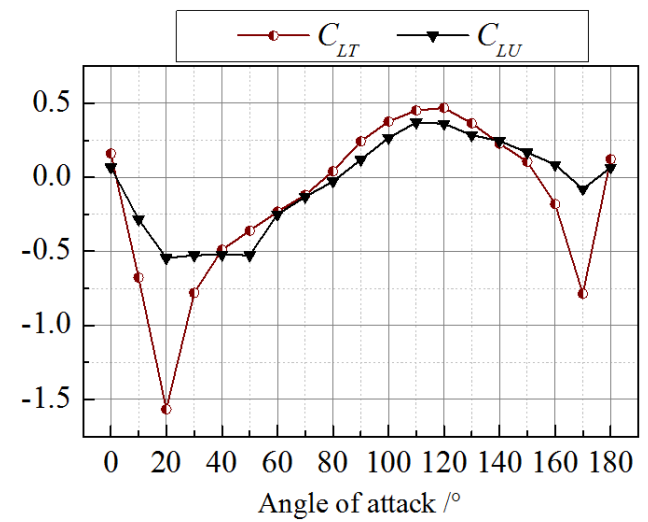

Figure 24. Lift coefficient in uniform flow and turbulent flow.

\subsection{Effect of Turbulence on the Aerodynamic}

Figures 23 and 24 show the aerodynamic test results of iced conductor, the icing shape coefficients of which are $\lambda_{X_{+}}=1.4$, with the average wind speed of $11 \mathrm{~m} / \mathrm{s}$ in uniform flow or turbulent flow. It can be seen that the variation of the iced conductor's aerodynamic coefficients is greater under the action of turbulent flow. The curve of drag coefficient has a trough at 20 degree. For the lift coefficient, the trough becomes more convex at the 20 degree or 170 degree, and the absolute value increases from 0.5 to 1.5 . The above results shows that the aerodynamic characteristics of the iced conductor changes more obviously under the action of turbulent flow and the turbulent flow is unfavorable for iced conductor's stability.

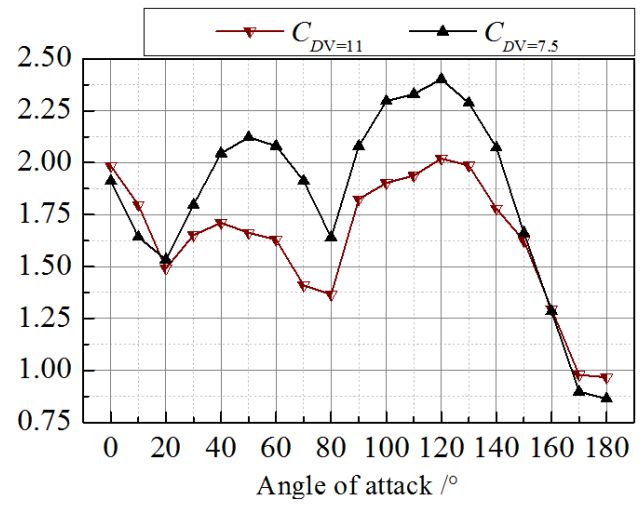

Figure 25. Drag coefficient for different wind speed in uniform.

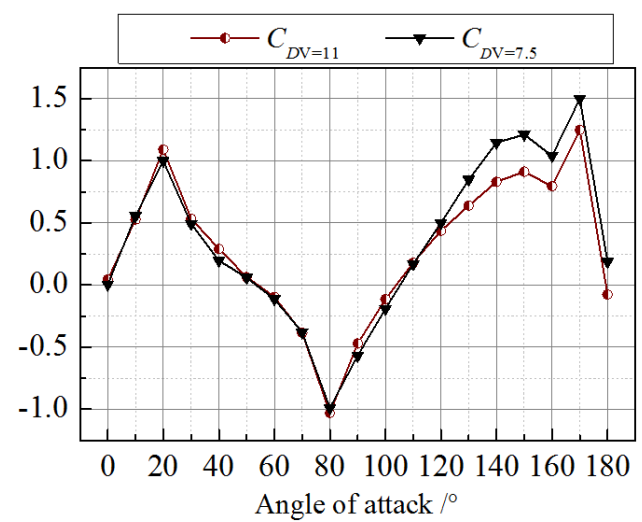

Figure 26. Lift coefficient for different wind speed in uniform flow.

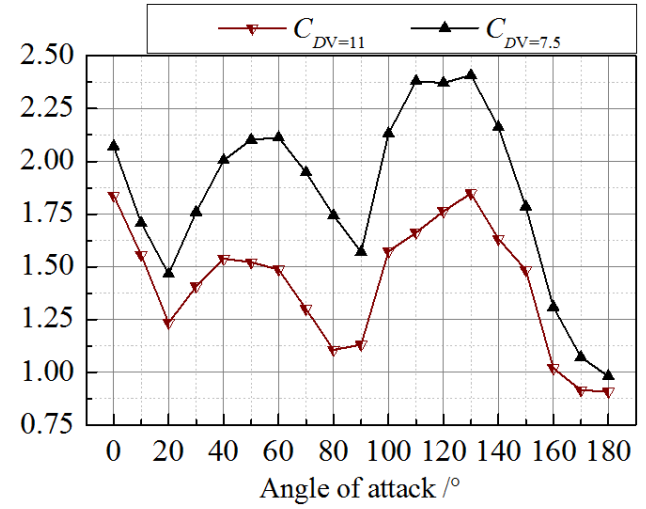

Figure 27. Drag coefficient for different wind speed in turbulent flow.

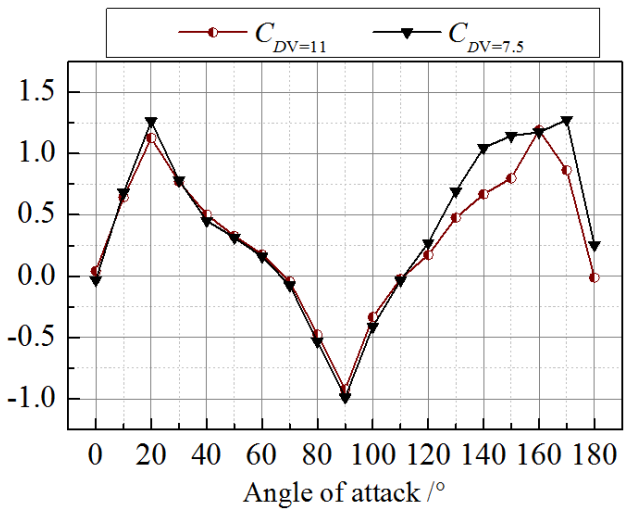

Figure 28. Lift coefficient for different wind speed in turbulent flow.

\subsection{Effect of Average Wind Speed on the Aerodynamic}

Figures 25 and 26 show the aerodynamic test results of iced conductor with the icing shape coefficient of $\lambda_{G+}=1.4$ in uniform flow considering different average wind speed $(U=7.5 \mathrm{~m} / \mathrm{s}$ or $U=11 \mathrm{~m} / \mathrm{s})$. It can be seen that the wind speed has much effect on the drag coefficient. The maximum value of the drag coefficient for $U=7.5 \mathrm{~m} / \mathrm{s}$ is larger than that of $U=11 \mathrm{~m} / \mathrm{s}$. The minimum value of the drag coefficient for $U=7.5 \mathrm{~m} / \mathrm{s}$ is less than that of $U=11 \mathrm{~m} / \mathrm{s}$. Meanwhile, it also can be seen from Figure 26 that the wind speed has little effect on the lift coefficient.

Figures 27 and 28 show the aerodynamic test results of iced conductor with the icing shape coefficient of $\lambda_{G+}=1.2$ in fluctuating wind for $U=11 \mathrm{~m} / \mathrm{s}$ and $U=7.5 \mathrm{~m} / \mathrm{s}$. And the 
turbulence intensity is $8 \%$. It can be seen that the wind speed has much effect on the drag coefficient. The drag coefficient for $U=11 \mathrm{~m} / \mathrm{s}$ is less than that of $U=7.5 \mathrm{~m} / \mathrm{s}$ at any wind attack angle. Furthermore, it can be also seen from Figure 28 that the wind speed has little effect on the lift coefficient.

\section{Conclusions}

In this paper, the force measurement test for aerodynamic characteristics of segment models of iced conductor is presented, and nine kinds of working conditions are included. We have studied the effects of icing types, icing thickness, turbulence intensity and average wind speed on the aerodynamic characteristics systematically. The main findings are summarized as follows:

1. The icing type has much effect on the aerodynamic characteristics. The aerodynamic characteristics of crescent icing shape appear to be more regular, while the fan and corona icing shapes have more peaks and troughs.

2. The shunt boards are indispensable to the test, which can guarantee the two-dimensional feature of flow field.

3. The aerodynamic instability of iced conductors is more remarkable in the turbulent flow, and the turbulent flow can promote galloping of iced conductors.

4. The wind speed has significant effect on drag coefficient, compared with lift coefficient.

\section{Acknowledgements}

The author gratefully acknowledges Prof. Hu Liang for his advice.

\section{References}

[1] Electric Power Research Institute (EPRI), Transmission Line Reference Book, Wind-Induced Conductor Motion, Palo Alto, California, 1979.

[2] A. M. Loredo-Souza and A. G. Davenport, "A novel approach for wind tunnel modelling of transmission lines", Journal of Wind Engineering and Industrial Aerodynamics, vol. 89, no. 11-12, pp. 1017-1029, 2001

[3] R. L. Wardlaw, K. R. Cooper, R. G. Ko, et al, "Wind tunnel and analytical investigations into the aeroelastic behaviour of bundled conductors", IEEE Transactions on Power Apparatus \& Systems, vol. 94, no. 2, pp. 642-654, 1975.

[4] D. S. Weaver and I. Veljkovic, "Vortex shedding and galloping of open semi-circular and parabolic cylinders in cross-flow",
Journal of Fluids and Structures Fluid-Structure and Flow-Acoustic Interactions involving Bluff Bodies, vol. 21, no. 1, pp. 65-74, 2005.

[5] Nakamura Y, "On the aerodynamic mechanism of torsional flutter of bluff structures", Journal of Sound \& Vibration, vol. 67, no. 2, pp. 163-177, 1979.

[6] G. Alonso and J. Meseguer, "A parametric study of the galloping stability of two-dimensional triangular cross-section bodies", Journal of Wind Engineering and Industrial Aerodynamics, vol. 94, no. 4, pp. 241-253, 2006.

[7] G. Alonso, E. Valero, and J. Meseguer, "An analysis on the dependence on cross section geometry of galloping stability of two-dimensional bodies having either biconvex or rhomboidal cross sections", European Journal of Mechanics-B/Fluids, vol. 28, no. 2, pp. 328-334, 2009.

[8] P. H. Fleming and N. Popplewell, "Wind Tunnel Studies on the Galloping of Lightly-Iced Transmission Lines", in Proceedings of the ASME 2010 3rd Joint Us-European Fluids Engineering Summer Meeting Collocated and 8th International Conference on Nanochannels, Microchannels, and Minichannels (FEDSM-ICNMM2010-30808), Montreal, Canada, 2010.

[9] Bo Yan, Xiaohui Liu, Xin Lv et al. "Investigation into galloping characteristics of iced quad bundle conductors", Journal of Vibration \& Control, vol. 22, no. 4, pp. 1-23, 2014.

[10] F. Gandia, J. Meseguer, and A. Sanzandrés, "Static and Dynamic Experimental Analysis of the Galloping Stability of Porous H-Section Beams", The scientific world journal, vol. 2014, Article ID 746826, 9 pages, 2014.

[11] Lou W, Yu J, Jiang X, et al. "Stability evaluation and aerodynamic damping study on three-degree-of-freedom coupled galloping of iced conductors", China Civil Engineering Journal, vol. 50, no. 2, pp. 55-64, 2017.

[12] Mingzhen LI, Hongliang ZHU, Hanlin LI et al. "Wind Tunnel Test of Drag Reduced Overhead Conductor", Wire \& Cable, no. 5, pp. 9-12, 2017.

[13] Wu C, Yan B, Huang G et al. "Wake-induced oscillation behaviour of twin bundle conductor transmission lines", Royal Society Open Science, vol. 5, no. 6, pp. 180011-180033, 2018.

[14] Yi Y, Cheng H, Xinxin W, "Wind Tunnel Tests on Aerodynamic Characteristics of two types of Iced Conductors with Elastic Support", IOP Conference Series Earth and Environmental Science, vol. 108, no. 5, pp. 1-5, 2018.

[15] Sun Ying, Wu Yue, and Cao Zhenggang, "Guide for building wind tunnel experiment", Beijing: China building industry press, 2011

[16] J. Franke and W. Frank, "Large eddy simulation of the flow past a circular cylinder at $\mathrm{Re}=3900$ ", Journal of Wind Engineering and Industrial Aerodynamics, vol. 90, no. 10, pp. 1191-1206, 2002. 